\title{
UNVEILING THE BOXY BULGE AND BAR OF THE ANDROMEDA SPIRAL GALAXY
}

\author{
Rachael L. Beaton, ${ }^{1}$ Steven R. Majewski, ${ }^{1}$ Puragra Guhathakurta, ${ }^{2}$ Michael F. Skrutskie, ${ }^{1}$ Roc M. Cutri, ${ }^{3}$ \\ John Good, ${ }^{3}$ Richard J. Patterson, ${ }^{1}$ E. Athanassoula, ${ }^{4}$ and Martin Bureau ${ }^{5}$ \\ Received 2006 May 9; accepted 2007 February 14; published 2007 March 7
}

\begin{abstract}
A new, $2.8 \operatorname{deg}^{2} J, H, K_{s}$ near-infrared (NIR) survey from the 2MASS 6X program across the extent of the optical disk of the Andromeda (M31) galaxy provides a clear view of the M31 center almost completely unfettered by dust extinction and reveals a high-contrast bulge with very boxy isophotes dominating the NIR light to a semimajor axis of $\sim 700^{\prime \prime}(2.6 \mathrm{kpc})$. The inner bulge $\left(\$ 50^{\prime \prime}\right)$ isophotes are relatively circular but show some twisting. Beyond this, (1) the M31 bulge ellipticity increases, (2) its position angle is constant at $\sim 50^{\circ}$, or about $10^{\circ}$ higher than the position angle of the M31 disk, and (3) its boxiness increases to a degree ( 3\%-4\%) comparable to other renowned examples of boxy bulges observed in the NIR. In a companion paper, self-consistent $N$-body simulations of a classical bulge plus a bar with a boxy bulge are shown to reproduce the observed NIR M31 features presented here. Beyond the boxy bulge region and nearly along the $40^{\circ}$ position angle of the disk a narrow ridge of NIR flux, which can be identified with the thin part of the bar, more or less symmetrically extends into the inner disk at semimajor axis radii of $700^{\prime \prime}-1200^{\prime \prime}$ or more. Little variation in the morphology or relative brightnesses of these various M31 structures is seen across the NIR bands (e.g., no color gradients are seen). These new data verify that M31 is a barred spiral galaxy like the Milky Way.
\end{abstract}

Subject headings: galaxies: bulges — galaxies: individual (M31) — galaxies: spiral — galaxies: structure — infrared: galaxies - Local Group

\section{INTRODUCTION}

For long after the discovery that spiral nebulae are "island universes," the Andromeda galaxy (M31) was thought to present a reasonable mirror image of our own Milky Way (MW). This morphological mirror, however, was clouded with the discovery of barred structure in the MW, revealed as both a gas dynamical structure (e.g., Binney et al. 1991) and a stellar feature (e.g., Blitz \& Spergel 1991; Weinberg 1992). More recent evidence (e.g., Parker et al. 2003, 2004; see also Newberg \& Yanny 2004) suggests that the MW's central bar may exert significant influence, or at least account for observable stellar density asymmetries, to large distances from the Galactic midplane.

Although there have been isolated lines of evidence supporting the existence of a bar in M31, its existence has remained uncertain. Fifty years ago Lindblad (1956) first interpreted the shape of the inner M31 isophotes as an indication that M31 has a triaxial bulge consistent with "a barred spiral structure." Stark (1977) attempted to match the observed isophotal twists and overall optical surface brightness distribution within $2 \mathrm{kpc}$ with a one-parameter family of triaxial models and also concluded that misalignment between the bulge and disk isophotes required a central bar. This notion was supported by evidence from gas dynamics (Stark \& Binney 1994; Berman 2001; Berman \& Loinard 2002). Although the Kent (1983) optical imaging did not identify the previously suggested misalignment of the bulge and disk isophotes, the data showed some degree of boxiness to the bulge isophotes (revealed by a nonzero fourth-order harmonic

\footnotetext{
${ }^{1}$ Department of Astronomy, University of Virginia, Charlottesville, VA 22904-4325; rlb9n@virginia.edu, srm4n@virginia.edu, mfs4n@virginia.edu, rjp0i@virginia.edu.

${ }^{2}$ UCO/Lick Observatory, Department of Astronomy and Astrophysics, University of California, Santa Cruz, CA 95064; raja@ucolick.org.

${ }^{3}$ Infrared Processing and Analysis Center, Caltech, Pasadena, CA 91125; roc@ipac.caltech.edu, jeg@ipac.caltech.edu.

${ }^{4}$ Observatoire de Marseille, 13248 Marseille Cedex 04, France; lia@oamp.fr.

${ }^{5}$ Sub-Department of Astrophysics, University of Oxford, Denys Wilkinson Building, Keble Road, Oxford OX1 3RH, UK; bureau@astro.ox.ac.uk.
}

in isophotal shapes) and provide a compelling implication of a more complicated central M31. However, all previous optical studies of M31 have been substantially hindered by dust contamination in this highly inclined $\left(i=77.5^{\circ}\right)$ system.

It has long been known that observations in the near-infrared (NIR), where the effects of dust are mitigated, can reveal central galaxy structures hidden at shorter wavelengths, including multiple nuclei, bars, and boxy/peanut-shaped bulges (e.g., Hackwell \& Schweizer 1983; Scoville et al. 1988; Telesco et al. 1991; Majewski et al. 1993; Quillen et al. 1997). The central regions of highly inclined systems are particularly challenging to study, but especially valuable for understanding the frequency of boxy/ peanut morphologies. Despite several surveys of highly inclined galaxies (e.g., Shaw 1993; Lütticke et al. 2000; Bureau et al. 2006), perhaps less than 100 have been studied in the NIR. This includes the MW, for which a clear portrait of its central, barlike structure has been revealed by the distribution of carbon stars in the Two Micron All Sky Survey (2MASS) point source catalog (Skrutskie et al. 2001; Cole \& Weinberg 2002). Comparable studies of M31 have not been previously available because of the combined need for greater depth over a large field of view. Such a view is now provided by deep scans of M31 using the 2MASS North facility.

\section{THE 6X OBSERVATIONS OF M31}

Near the end of the normal 2MASS observing period, longexposure observations were taken of a number of special target regions, including M31, during idle times in the regular operation of the survey. These observations, taken with exposures 6 times longer than the nominal 2MASS observations and referred to as the "6X observations," used the same freeze-frame scanning as the main survey. ${ }^{6} \mathrm{M} 31$ was observed using $1^{\circ}$ long scans similar to those used for 2MASS calibration field observations. A telescope command error produced coverage gaps

\footnotetext{
${ }^{6}$ See http://www.ipac.caltech.edu/2mass/releases/allsky/doc/ for a description of the 2MASS $6 \mathrm{X}$ survey.
} 


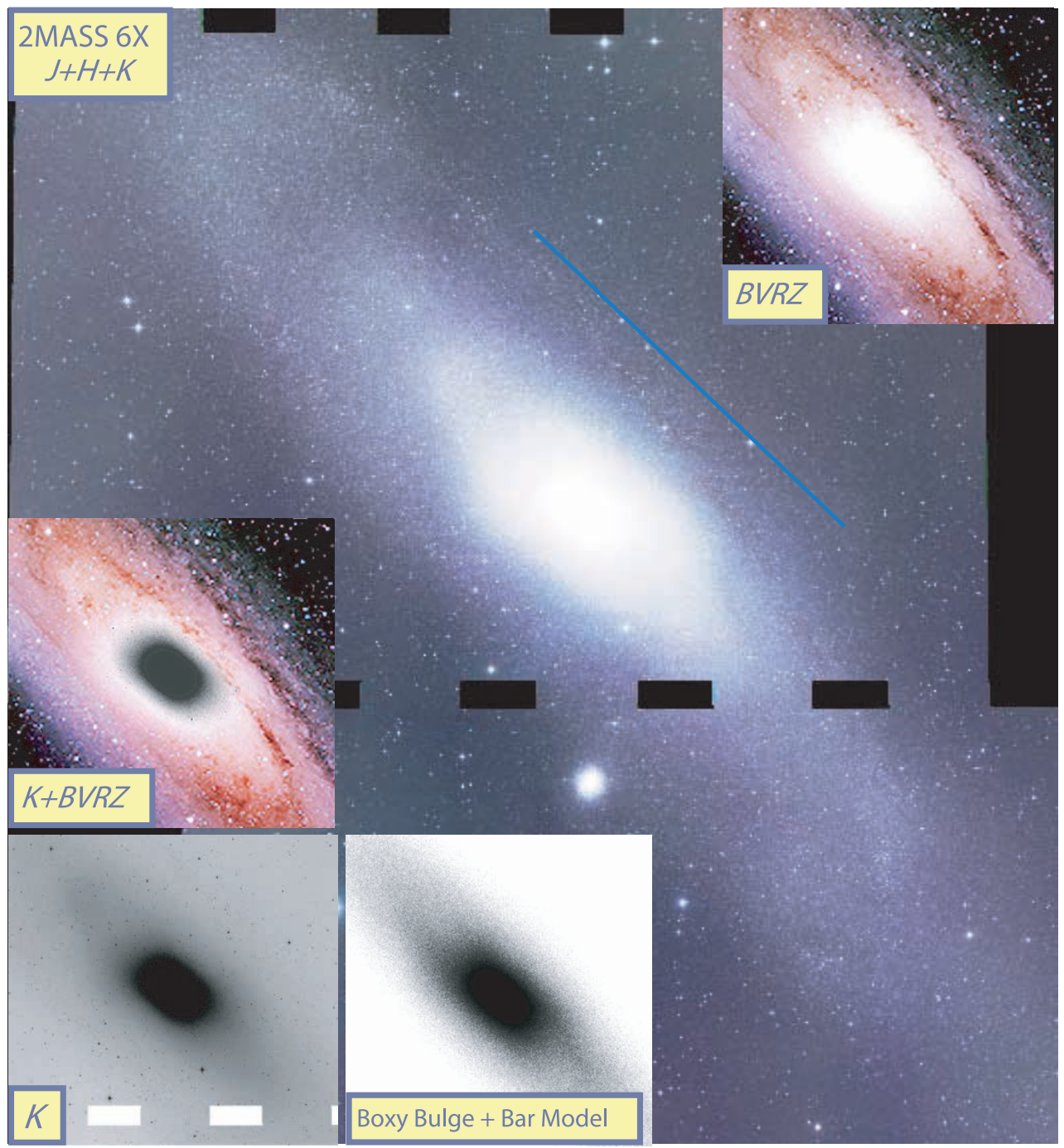

FIG. 1.-Central image is a color composite using the $J, H$, and $K_{s}$ images of M31 from the 2MASS 6 X observations. The line is $2400 "$ ( 8 kpc) long (parallel to, and the length of, the thin bar feature). The contributing $K_{s}$ image is shown separately in the lower left inset. The upper right inset is a color composite from the optical $B, V, R, z$ bands. An additional inset shows an overlap of the $K_{s}$ and $B, V, R, z$ images to show the relative positions of features. We also include a boxy bulge plus bar model matching the system from AB06. Insets are $30 \%$ smaller than the central image.

of a few $\operatorname{arcmin}^{2}$ in the $2.8 \mathrm{deg}^{2} \mathrm{M} 316 \mathrm{X}$ mosaic. These gaps (visible in Fig. 1) do not affect the work presented here.

We adopt the standard reduction of the 6X M31 data provided by the 2MASS Project, which used a slightly modified version of the automated data reduction pipeline utilized to process the original survey data. The final output $6 \mathrm{X}$ Atlas Images are identical in format to those from the original survey. Composite $J, H$, and $K_{s}$ mosaics were constructed from the individual 6X M31 Atlas Images using the Montage ${ }^{7}$ software package. Montage matches the background levels of all images by applying an additive offset and gradient correction that is computed using a least-squares fit to the mean intensity offsets of all pixels in the overlapping regions between each pair of images. Corrections were applied to the images to match the photometric zero points between adjacent 6X scans. Mean "sky" levels determined from the peak of the histogram of pixel values in the outer image (beyond the apparent disk of M31 defined by the ring of $\mathrm{H}$ II regions readily apparent in the

${ }^{7}$ See http://montage.ipac.caltech.edu/.
Galaxy Evolution Explorer images of M31; see, e.g., Thilker et al. 2005) were subtracted from each the $J, H$, and $K_{s}$ images to adjust pixel values to an approximately linear representation of M31 flux.

\section{THE NEAR-INFRARED STRUCTURE OF M3}

A boxy, high surface brightness bulge is the most obvious M31 feature in the 6X images, and this significantly distinguishes M31's NIR morphology from that seen in the optical (Fig. 1). Apart from slightly more dust modulation in $J$ (affecting the $J-K_{s}$ color by only a few to $10 \%$ at the dust lanes northwest of the bulge), the basic character of M31 is the same across the NIR bands, most evidently in the similarity of the high-contrast region of the bulge with its $\sim 700^{\prime \prime}$ major axis radius and, at fainter surface brightness, the appearance of a thin ridge of NIR light just beyond this bulge nearly along the disk major axis.

To quantify this morphology, ellipses were fit to the isophotal contours in each the $J, H$, and $K_{s}$ bands using the ELLIPSE 


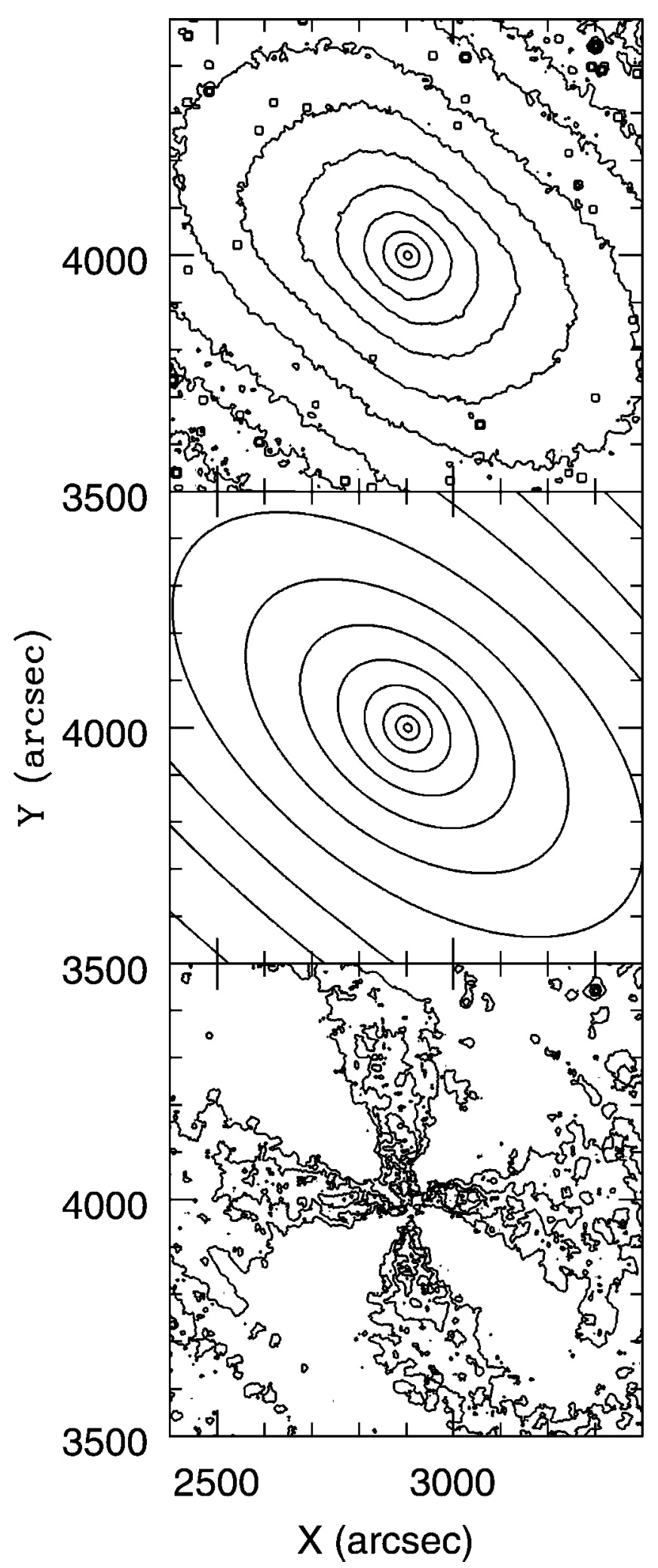

FIG. 2.-Top: Contour plot for the central $K_{s}$ band image. Middle: Best-fit ellipses to the $K_{s}$ data. Bottom: Residual plots created by subtracting the bestfitting ellipse from the $K_{s}$ data. The contours in all three panels have uniform logarithmic spacing. The pixel coordinates are pixel values in the $6 \mathrm{X}$ image; the image scale is $1^{\prime \prime}$ pixel $^{-1}$. The $X$ and $Y$ coordinates are aligned with the right ascension and declination axes.

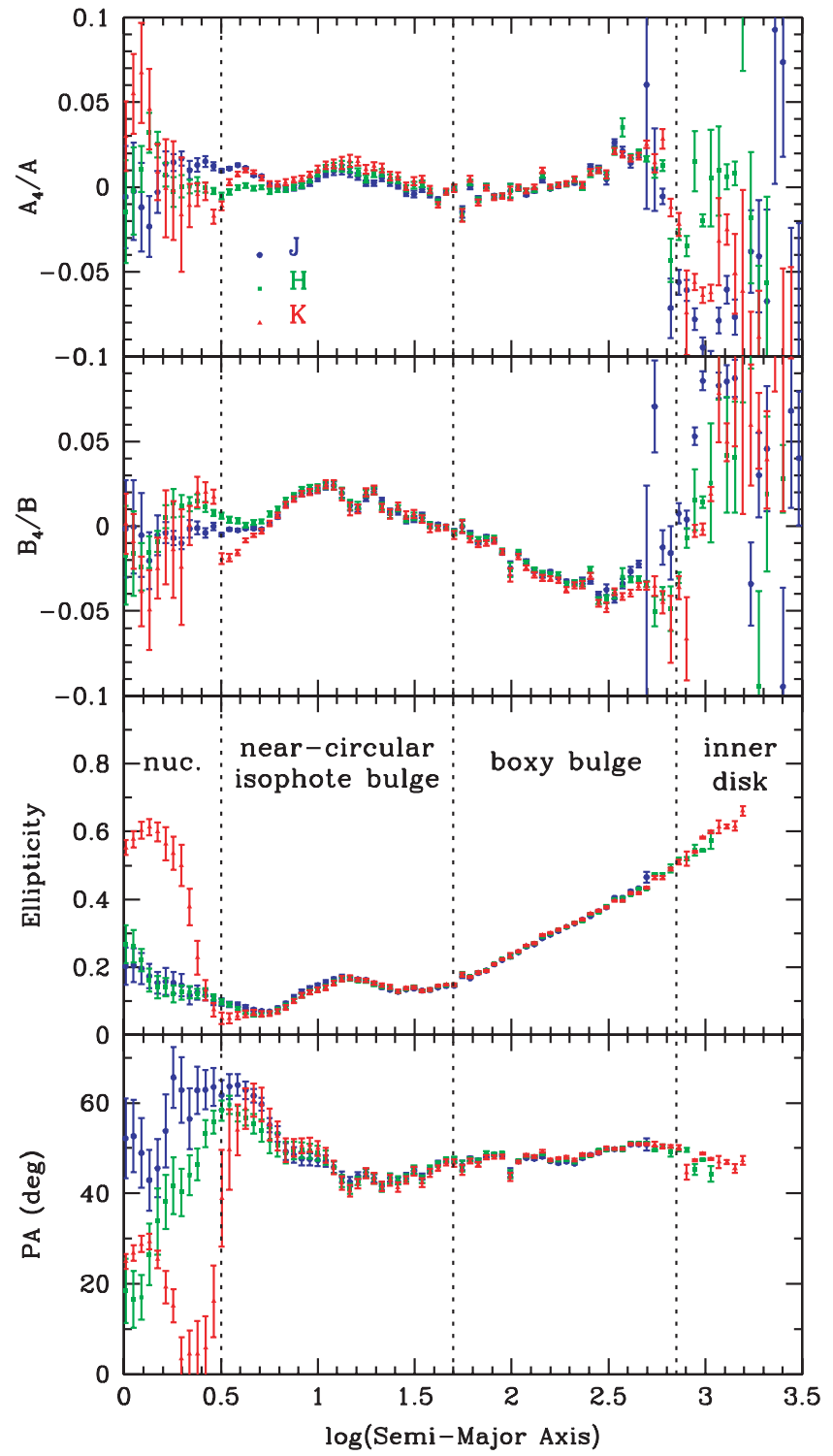

FIG. 3. $-A_{4} / A, B_{4} / B$, ellipticity and P.A. of M31 as a function of semimajor axis (in arcseconds) as measured in the $J, H$, and $K_{s}$ bands (blue, green, and red points, respectively).

package in $\operatorname{IRAF}^{8}$ (Fig. 2). This code uses the techniques of Jedrzejewski (1987) to fit a Fourier series to concentric galactic isophotes. The fourth-order coefficients $\left(A_{4}\right.$ and $\left.B_{4}\right)$ of the bestfit Fourier series are a useful descriptor of the deviation of the isophotes from true ellipses, from "boxy" $\left(B_{4}<0, A_{4}>0\right)$ to "disky" $\left(B_{4}>0, A_{4}<0\right)$. The strong boxiness of the M31 bulge is evident not only by its Fourier decomposition (Fig. 3) but by the difference between the galaxy isophotes and their corresponding best-fitting ellipses (Fig. 2). The ELLIPSE algorithm also determines the position angle (P.A.) of the major axis of each isophote and its ellipticity $(e=1-b / a)$, shown in Figure 3.

Based on trends in Figures 2-3, we discriminate four observational regions by semimajor axis radius within the inner $\sim 3300^{\prime \prime}$ of M31 (see divisions in Fig. 3):

1. Moderately circular isophotes dominate the central $<3$ ".

${ }^{8}$ IRAF (the Image Reduction and Analysis Facility) is distributed by the National Optical Astronomy Observatories. 
This nucleus was shown to be an independent M31 feature (Light et al. 1974), so the $<3$ " characteristics do not bear on the bulge morphology. In any case, because of the $1^{\prime \prime}$ pixel sampling, the P.A. and Fourier decomposition here have little meaning.

2. A region with near-circular isophotes $\left(3^{\prime \prime}-50^{\prime \prime}\right)$ surrounds the nucleus and resembles a classical bulge with low ellipticity. The primary structural characteristic of this radial zone is a $20^{\circ}$ change in P.A. This isophotal twisting is evident by the slight kinking of the innermost parts of the "Maltese cross" pattern in the bottom panel of Figure 2.

3. The boxy bulge $\left(\sim 50^{\prime \prime}-700^{\prime \prime}\right)$ exhibits distinctly negative values of $B_{4} / B$ and a steadily increasing ellipticity with radius (at constant P.A.). The strongest boxiness, at $\sim 300^{\prime \prime}$, is evident by the width and amplitude of the Maltese cross arms at this point.

4. Finally, a transition to the inner disk $\left(>700^{\prime \prime}\right)$ is demarcated by the shift to disky Fourier components and by the lower surface brightness evident in Figure 1. Because of the lower surface brightness and partial coverage, the P.A. and ellipticity are less reliably measured here. However, an obvious feature in this radial zone is the narrow bar structure protruding from both ends of the boxy bulge, shifted to nearly the disk P.A., and extending to a radius of $\sim 1200^{\prime \prime}(4 \mathrm{kpc}$ ) or more (see also Athanassoula \& Beaton 2006, hereafter AB06).

Several trends hold throughout the bulge/bar regions just described: (1) The three infrared bands closely track one another in morphology and relative brightness, so that no radial color gradients are evident outside the nucleus (this color uniformity is evident by the nearly uniform whiteness of the central structures in the color composite in Fig. 1), (2) the center of the bulge isophotes for different NIR bands are coincident at the center of the optical disk, (3) the primary P.A. of the bulge, about $50^{\circ}$, is about $10^{\circ}$ higher than the P.A. of the outer disk (as had been found by Lindblad), and (4) the 3\%-4\% boxiness achieved by M31's bulge is comparable to the most "classical" examples of boxy (although not peanut-shaped) bulges seen in other NIR studies (e.g., Shaw 1993; Bureau et al. 2006).

\section{DISCUSSION}

This new large-scale infrared mapping of M31 vividly highlights the morphology of its bulge, definitively confirms earlier suggestions from optical studies ( $§ 1)$ of a boxy inner structure, and permits high signal-to-noise ratio measurements of bulge shape without interference from variable dust extinction (Fig. 3).
M31 clearly twins the MW (Hauser et al. 1990; Weiland et al. 1994) - as well as a large fraction (45\%, according to Lütticke et al. 2000) of other highly inclined disk galaxies - in having a boxy inner structure that is made more evident by infrared imaging. Indeed, $K$-band images by Shaw (1993) and Bureau et al. (2006) of nearly 30 edge-on systems with a boxy/peanut bulge all display residual or unsharp-masked maps with cross patterns resembling that observed here in M31 (Fig. 2).

$\mathrm{N}$-body simulations show that bars can thicken considerably, forming a boxy/peanut structure (Combes et al. 1990). Thus, boxy bulges are now recognized to be parts of bars, namely, the vertically thick part, with the thin part of the bar extending to larger radii, beyond the boxy/peanut part (Athanassoula 2005). This is clearly demonstrated by the MW, which appears to have a boxy bulge similar to the one we observe in M31 (Binney et al. 1997; Bissantz \& Gerhard 2002). In a companion paper, AB06 used fully self-consistent $N$-body simulations to model the inner structure of M31. They reproduce well the NIR isophotal shapes and light profiles along cross sections parallel to the major axis and account for the pseudo-ring at 50' as an outer resonant ring, commonly found in barred galaxies. In this model, the bar has a boxy bulge, and its vertically thin part extends well past the 700" boxy bulge.

The 6X images show clear evidence for this "thin bar" extending to at least $\sim 1200^{\prime \prime}$ (4 kpc; Fig. 1). From the presence of this feature, together with the successful modeling of the images presented here by a self-consistent $N$ body, barred galaxy simulation as well as the numerous other examples of models and data indicating the close connection between bars and boxy bulges, it may be safely concluded that M31 is a barred spiral galaxy.

This publication uses data products from the Two Micron All Sky Survey, a joint project of the University of Massachusetts and Infrared Processing and Analysis Center/California Institute of Technology, funded by the National Aeronautics and Space Administration (NASA) and National Science Foundation (NSF). We appreciate support by NSF grant AST 0307851 (SRM), NASA/JPL contract 1228235 (S. R. Majewski), NASA/JPL contract 1234021 (M. F. Skrutskie), and NSF grants AST 03-07966 and AST 05-07483 and NASA/STScI grants GO-10265.02 and GO-10134.02 to P. Guhathakurta. R. L. Beaton was supported by a Harrison Undergraduate Research Award from the University of Virginia Center for Undergraduate Research. This work was also supported by the F. H. Levinson Fund of the Peninsular Community Foundation.

\section{REFERENCES}

Athanassoula, E. 2005, MNRAS, 358, 1477

Athanassoula, E., \& Beaton, R. L. 2006, MNRAS, 370, 1499 (AB06)

Berman, S. 2001, A\&A, 371, 476

Berman, S., \& Loinard, L. 2002, MNRAS, 336, 477

Binney, J., Gerhard, O., \& Spergel, D. 1997, MNRAS, 288, 365

Binney, J., Gerhard, O. E., Stark, A. A., Bally, J., \& Uchida, K. I. 1991, MNRAS, 252, 210

Bissantz, N., \& Gerhard, O. 2002, MNRAS, 330, 591

Blitz, L., \& Spergel, D. N. 1991, ApJ, 379, 631

Bureau, M., Aronica, G., Athanassoula, E., Dettmar, R.-J., Bosma, A., \& Freeman, K. C. 2006, MNRAS, 370, 753

Cole, A. A., \& Weinberg, M. D. 2002, ApJ, 574, L43

Combes, F., Debbasch, F., Friedli, D., \& Pfenniger, D. 1990, A\&A, 233, 82

Hackwell, J. A., \& Schweizer, F. 1983, ApJ, 265, 643

Hauser, M. G., et al. 1990, NASA photograph G90

Jedrzejewski, R. I. 1987, MNRAS, 226, 747

Kent, S. M. 1983, ApJ, 266, 562

Light, E. S., Danielson, R. E., \& Schwarzschild, M. 1974, ApJ, 194, 257

Lindblad, B. 1956, Stockholm Obs. Ann., 2,

Lütticke, R., Dettmar, R.-J., \& Pohlen, M. 2000, A\&A, 362, 435
Majewski, S. R., Hereld, M., Koo, D. C., Illingworth, G. D., \& Heckman, T. M. 1993, ApJ, 402, 125

Newberg, H. J., \& Yanny, B. 2004, BAAS, 36, 1582

Parker, J. E., Humphreys, R. M., \& Beers, T. C. 2004, AJ, 127, 1567

Parker, J. E., Humphreys, R. M., \& Larsen, J. A. 2003, AJ, 126, 1346

Quillen, A. C., Kuchinski, L. E., Frogel, J. A., \& Depoy, D. L. 1997, ApJ, 481,179

Scoville, N. Z., Matthews, K., Carico, D. P., \& Sanders, D. B. 1988, ApJ, 327, L61

Shaw, M. 1993, MNRAS, 261, 718

Skrutskie, M. F., Reber, T. J., Murphy, N. W., \& Weinberg, M. D. 2001, BAAS, 33, 1437

Stark, A. A. 1977, ApJ, 213, 368

Stark, A. A., \& Binney, J. 1994, ApJ, 426, L31

Telesco, C. M., Joy, M., Dietz, K., Decher, R., \& Campins, H. 1991, ApJ, 369,135

Thilker, D. A., et al. 2005, ApJ, 619, L67

Weiland, J. L., et al. 1994, ApJ, 425, L81

Weinberg, M. D. 1992, ApJ, 384, 81 